\title{
Response-Guided Telaprevir Combination Treatment for Hepatitis C Virus Infection
}

\author{
Kenneth E. Sherman, M.D., Ph.D., Steven L. Flamm, M.D., Nezam H. Afdhal, M.D., David R. \\ Nelson, M.D., Mark S. Sulkowski, M.D., Gregory T. Everson, M.D., Michael W. Fried, M.D., \\ Michael Adler, M.D., Ph.D., Hendrik W. Reesink, M.D., Ph.D., Marie Martin, Ph.D., Abdul J. \\ Sankoh, Ph.D., Nathalie Adda, M.D., Robert S. Kauffman, M.D., Ph.D., Shelley George, M.D., \\ Christopher I. Wright, M.D., Ph.D., and Fred Poordad, M.D. for the ILLUMINATE Study \\ Team \\ From the Division of Digestive Diseases, University of Cincinnati College of Medicine, Cincinnati \\ (K.E.S.); the Division of Hepatology, Northwestern University Medical School, Chicago (S.L.F.); \\ the Division of Gastroenterology and Hepatology, Beth Israel Deaconess Medical Center, Boston \\ (N.H.A.); the Section of Hepatology, University of Florida, Gainesville (D.R.N.); the Viral Hepatitis \\ Center, Johns Hopkins University School of Medicine, Baltimore (M.S.S.); the Section of \\ Hepatology, Division of Gastroenterology and Hepatology, University of Colorado School of \\ Medicine Denver, Aurora (G.T.E.); the UNC Liver Center, University of North Carolina, Chapel Hill \\ (M.W.F.); the Department of Gastroenterology and Hepatopancreatology, Erasme Hospital \\ Brussels, Brussels (M.A.); the Department of Hepatology, Academic Medical Center of the \\ University of Amsterdam, Amsterdam (H.W.R.); Vertex Pharmaceuticals, Cambridge, MA (M.M., \\ A.J.S., N.A., R.S.K., S.G., C.I.W.); and the Department of Medicine, Cedars-Sinai Medical \\ Center, Los Angeles (F.P.)
}

\section{Abstract}

BACKGROUND—Patients with chronic infection with hepatitis C virus (HCV) genotype 1 often need 48 weeks of peginterferon-ribavirin treatment for a sustained virologic response. We designed a noninferiority trial (noninferiority margin, $-10.5 \%$ ) to compare rates of sustained virologic response among patients receiving two treatment durations.

METHODS-We enrolled patients with chronic infection with HCV genotype 1 who had not previously received treatment. All patients received telaprevir at a dose of $750 \mathrm{mg}$ every 8 hours, peginterferon alfa-2a at a dose of $180 \mu \mathrm{g}$ per week, and ribavirin at a dose of 1000 to $1200 \mathrm{mg}$ per day, for 12 weeks (T12PR12), followed by peginterferon-ribavirin. Patients who had an extended rapid virologic response (undetectable HCV RNA levels at weeks 4 and 12) were randomly assigned after week 20 to receive the dual therapy for 4 more weeks (T12PR24) or 28 more weeks (T12PR48). Patients without an extended rapid virologic response were assigned to T12PR48.

RESULTS-Of the 540 patients, a total of 352 (65\%) had an extended rapid virologic response. The overall rate of sustained virologic response was $72 \%$. Among the 322 patients with an extended rapid virologic response who were randomly assigned to a study group, 149 (92\%) in the T12PR24 group and 140 (88\%) in the T12PR48 group had a sustained virologic response (absolute difference, 4 percentage points; 95\% confidence interval, -2 to 11), establishing

Copyright (@) 2011 Massachusetts Medical Society.

Address reprint requests to Dr. Sherman at the Division of Digestive Diseases, University of Cincinnati College of Medicine, 231 Albert Sabin Way, Cincinnati, OH 45267-0595, or at shermake@ucmail.uc.edu.

${ }^{*}$ The ILLUMINATE (Illustrating the Effects of Combination Therapy with Telaprevir) study investigators are listed in the Supplementary Appendix, available at NEJM.org.

Disclosure forms provided by the authors are available with the full text of this article at NEJM.org. 
noninferiority. Adverse events included rash (in 37\% of patients, severe in 5\%) and anemia (in $39 \%$, severe in 6\%). Discontinuation of all the study drugs was based on adverse events in $18 \%$ of patients overall, as well as in $1 \%$ of patients (all of whom were randomly assigned) in the T12PR24 group and $12 \%$ of the patients randomly assigned to the T12PR48 group $(\mathrm{P}<0.001)$.

CONCLUSIONS-In this study, among patients with chronic HCV infection who had not received treatment previously, a regimen of peginterferon-ribavirin for 24 weeks, with telaprevir for the first 12 weeks, was noninferior to the same regimen for 48 weeks in patients with undetectable HCV RNA at weeks 4 and 12, with an extended rapid virologic response achieved in nearly two thirds of patients. (Funded by Vertex Pharmaceuticals and Tibotec; ILLUMINATE ClinicalTrials.gov number, NCT00758043.)

Chronic infection with hepatitis c virus (HCV) represents a serious health issue for nearly 200 million infected persons worldwide. ${ }^{1}$ Achievement of a sustained virologic response may be associated with improved long-term clinical outcomes, including increased survival. ${ }^{2,3}$ In patients infected with HCV genotype 1, 48 weeks of treatment with peginterferon alfa and ribavirin results in a rate of sustained virologic response of 40 to $50 \% .^{4,5}$

Telaprevir administered in combination with peginterferon and ribavirin has led to high rates of sustained virologic response in phase 2 and phase 3 trials involving patients with HCV genotype 1 infection, who have not previously received treatment, in the United States and Europe ${ }^{6-8}$ Telaprevir is an orally bioavailable inhibitor of the nonstructural 3/4A (NS3/4A) $\mathrm{HCV}$ protease. Phase 2 data have suggested that a high proportion of patients have a rapid decline in viral levels during early treatment stages and that a response-guided treatment regimen based on viral response may permit a shorter treatment duration while preserving high rates of sustained virologic response. Such a strategy may decrease the risk of exposure of patients to not only potential side effects from telaprevir but also to the well-characterized adverse events associated with the 48 -week use of peginterferon and ribavirin.

In this multinational, randomized study, we assessed the efficacy and safety of responseguided therapy consisting of a three-drug regimen of telaprevir, peginterferon alfa- $2 \mathrm{a}$, and ribavirin in patients with chronic $\mathrm{HCV}$ genotype 1 infection who had not previously received treatment. The primary goal was to assess the noninferiority of a 24 -week versus a 48-week telaprevir-based regimen among patients who had an extended rapid virologic response (undetectable HCV RNA levels at weeks 4 and 12).

\section{METHODS \\ STUDY PATIENTS}

Eligible patients were enrolled at 74 sites in Belgium, the Netherlands, and the United States (including Puerto Rico). Inclusion criteria for enrollment were the presence of chronic infection with HCV genotype 1, indicated by a diagnosis at more than 6 months before the screening visit, with a detectable HCV RNA level at the visit, as well as no previous treatment for $\mathrm{HCV}$ infection; age between 18 and 70 years; seronegative test for hepatitis B virus and human immunodeficiency virus; an absolute neutrophil count of 1500 or more per cubic millimeter; a platelet count of 90,000 or more per cubic millimeter; and a hemoglobin level of $12 \mathrm{~g}$ or more per deciliter for female patients and $13 \mathrm{~g}$ or more per deciliter for male patients. All patients had undergone a liver biopsy within 1 year before the screening visit or underwent the procedure during the screening period unless a biopsy more than 1 year previously showed evidence of cirrhosis. Patients with hepatic decompensation, clinically significant liver disease from another cause, or active cancer within the previous 5 years (except treated basal-cell carcinoma) were excluded. 


\section{STUDY DESIGN AND CONDUCT}

All patients provided written informed consent. The study was approved by the institutional review boards of all study centers and was performed in accordance with Good Clinical Practice guidelines as defined by the International Conference on Harmonization and the Declaration of Helsinki. The study protocol and statistical analysis plan are available with the full text of this article at NEJM.org.

Vertex Pharmaceuticals and Tibotec funded the study. The academic principal investigator participated in study design and protocol development with the study sponsors, had unrestricted access to the data, prepared the first draft of the manuscript, and made the decision to submit the manuscript for publication. All authors reviewed and approved the final manuscript and assume responsibility for the accuracy and completeness of the data reported. An employee of Vertex Pharmaceuticals provided medical writing, editorial, and coordination services.

The ILLUMINATE (Illustrating the Effects of Combination Therapy with Telaprevir) trial was an open-label, randomized, phase 3 noninferiority trial in which the results of HCV RNA testing were double-blinded through week 24. All patients received triple therapy for 12 weeks, consisting of telaprevir (Vertex Pharmaceuticals) at a dose of $750 \mathrm{mg}$ orally every 8 hours, peginterferon alfa-2a (40 kD) (Pegasys, Roche) at a dose of $180 \mu \mathrm{g}$ injected subcutaneously once weekly, and ribavirin (Copegus, Roche) at a dose of $1000 \mathrm{mg}$ per day for patients weighing less than $75 \mathrm{~kg}$ or $1200 \mathrm{mg}$ per day for patients weighing $75 \mathrm{~kg}$ or more. After 12 weeks, all patients continued to receive peginterferon-ribavirin only.

\section{ASSESSMENT OF EFFICACY}

At study visits, the HCV RNA level was measured with the use of the COBAS TaqMan assay (Roche Molecular Systems, version 2.0), which has a lower limit of quantification of $25 \mathrm{IU}$ per milliliter and a lower limit of detection of approximately 10 to $15 \mathrm{IU}$ per milliliter.

After the week 20 visit, patients who had had an undetectable HCV RNA level at week 4 and week 12 (i.e., an extended rapid virologic response) were randomly assigned, in a 1:1 ratio, to stop treatment at week 24 (the T12PR24 group) or to continue peginterferonribavirin therapy through 48 weeks (the T12PR 48 group). Randomization was centrally managed, blocked, and stratified to optimize balance with regard to HCV genotype subtype (1a, 1b, or unknown) and self-reported race (black or nonblack). Patients who had not had an extended rapid virologic response were nonrandomly assigned to the T12PR48 group.

Upon the completion of treatment, all patients entered the follow-up phase, consisting of 48 weeks for the T12PR24 group and 24 weeks for the T12PR48 group. Patients who discontinued treatment before randomization were also followed for 24 weeks. All patients were assessed for a sustained virologic response at 24 weeks of follow-up and, in patients in the T12PR24 group, at 48 weeks of follow-up. All patients who completed the follow-up period were assessed for a response to treatment.

Virologic failure was defined as an HCV RNA level greater than 1000 IU per milliliter at week 4 , a decline from baseline by less than $2 \log _{10}$ units in the level of detectable $\mathrm{HCV}$ RNA at week 12, or a detectable HCV RNA level at any time between weeks 24 and 36. According to the study protocol, telaprevir was stopped in patients with an HCV RNA level greater than $1000 \mathrm{IU}$ per milliliter at week 4, and all study drugs were stopped in patients who had virologic failure at week 12 or between weeks 24 and 36. 
Samples for sequence-based virologic assessment of changes in the NS3/4A coding domain were collected at baseline. These were compared with samples obtained during the study for patients in whom HCV RNA did not become undetectable.

\section{SAFETY}

Planned safety evaluations included physical examinations, laboratory evaluation of key safety variables, and adverse-event reporting according to the Division of Acquired Immunodeficiency Syndrome (DAIDS) criteria for the assessment of severity. For adverse events not listed in the DAIDS criteria, severity assessment was based on protocol-defined criteria. Electrocardiography was performed at baseline. During the first 12 weeks of the study, investigators could discontinue the use of telaprevir (while continuing to administer peginterferon-ribavirin) if serious adverse events potentially attributable to telaprevir were observed. These included, but were not restricted to, rash and anemia. The use of erythropoietin or other hematopoietic growth factors was prohibited per the final amended protocol, as were reductions of telaprevir dose.

Planned reviews of safety data were conducted by an independent data and safety monitoring committee to evaluate the safety and tolerability of the study-drug regimens. A statistical group independent of the sponsors performed the analyses and safety-data preparation for each review.

\section{STATISTICAL ANALYSIS}

The primary efficacy variable was a sustained virologic response, defined as an undetectable HCV RNA level at the end of the treatment phase and 24 weeks after the last planned dose of study medication. The primary analysis was the estimation of the difference in sustained virologic response between patients who had extended rapid virologic response and were randomly assigned to receive T12PR24 versus T12PR48. The sample size was chosen to permit a noninferiority comparison between these two randomized subgroups with a predefined noninferiority margin of $-10.5 \%$. A sample size of 157 randomly assigned patients per study group was estimated to have $80 \%$ power to rule out the noninferiority of T12PR24 as compared with T12PR48 if the observed rate of sustained virologic response was $90 \%$.

\section{RESULTS}

\section{BASELINE CHARACTERISTICS OF THE STUDY PATIENTS}

A total of 540 patients received at least one dose of a study drug (Fig. 1) and represent the overall study population (Table 1). Black race was self-reported in 73 patients (14\%); 54 patients (10\%) identified themselves as Hispanic or Latino; and $3(1 \%)$ identified themselves as black and Hispanic or Latino. A total of 149 patients (28\%) had bridging fibrosis or cirrhosis. Genotype subtype 1a was predominant (388 patients [72\%]).

\section{EFFICACY AND NONINFERIORITY}

Overall, $72 \%$ (389 of 540) of the study patients had a rapid virologic response, defined as undetectable HCV RNA at week 4; 65\% (352 of 540) had an extended rapid virologic response. One hundred patients, including 30 who had an extended rapid virologic response, discontinued the study drugs owing to adverse events, withdrawal of consent, or other reasons. The overall rate of sustained virologic response was 72\% (Table 2). Of the 322 patients who had an extended rapid virologic response and a week 20 visit, 162 were randomly assigned to the T12PR24 group, of whom 149 (92\%) had a sustained virologic response; and 160 were randomly assigned to the T12PR48 group, of whom $140(88 \%)$ had a sustained virologic response. A total of 118 patients who had not had an extended rapid 
virologic response were assigned to the T12PR48 group, and 76 (64\%) had a sustained virologic response. Of the remaining 100 patients, who prematurely discontinued treatment before week 20, 23 (23\%) had a sustained virologic response.

The primary study objective was the noninferiority of the T12PR24 regimen to the T12PR48 regimen; specifically, the rate of sustained virologic response among patients with an undetectable HCV RNA level at weeks 4 and 12 who were randomly assigned to 24-week versus 48 -week treatment. The majority of patients $(99 \%)$ in the 24 -week group completed the treatment, as compared with $74 \%$ in the 48 -week group $(\mathrm{P}<0.001)$. The absolute difference in the rate of sustained virologic response between the T12PR24 group and the T12PR48 group was 4 percentage points (95\% confidence interval [CI], -2 to 11$)$. The lower limit of this $95 \%$ CI $(-2 \%)$ excluded the noninferiority margin of $-10.5 \%$.

Low rates of relapse were observed after the completion of treatment (6\% with T12PR24 and 3\% with T12PR48), with an overall relapse rate of $8 \%$ (Table 2). The HCV RNA level was undetectable at 72 weeks after the start of treatment in $70 \%$ (377 of 540) of patients overall, with an absolute difference of -0.5 percentage points between the T12PR 24 group $(87.0 \%)$ and the patients in the T12PR48 group who had had an extended rapid virologic response ( $87.5 \%)$ (95\% CI, -7.7 to 6.8$)$.

Rates of response in subgroups with host or baseline characteristics that have been historically associated with poor treatment outcome were also examined. Overall, $69 \%$ of patients with a high viral load ( $\$ 800,000$ IU per milliliter) had a sustained virologic response. Among those with an extended rapid virologic response, HCV RNA levels at baseline were not a predictor of a sustained virologic response, which was achieved in nearly $90 \%$ of patients with high viral load. Similarly, genotype subtype (1a vs. 1b) did not affect the treatment outcome, either in the overall population or among randomly assigned patients (Table 2). In all, 63\% of patients with bridging fibrosis or cirrhosis had a sustained virologic response. Among patients with an extended rapid virologic response and bridging fibrosis or cirrhosis, rates of sustained virologic response were 82\% in the T12PR24 group and $88 \%$ in the T12PR48 group (Table 2). This difference was not significant. Overall, $74 \%$ of white patients versus $60 \%$ of black patients had a sustained virologic response $(\mathrm{P}=0.02)$ (Table 2). Neither race nor ethnic group was associated with any decrease in the rate of sustained virologic response among the patients who had had an extended rapid virologic response; no significant differences were observed between the two randomized subgroups.

\section{VIROLOGIC FAILURE AND TELAPREVIR-RESISTANT VARIANTS}

Virologic failure during the treatment phase, including in patients who met the stopping rules and had detectable HCV RNA levels at the end of treatment, was uncommon, found in $8 \%$ of the overall population and in $2 \%$ of patients in the T12PR24 group and 3\% of the patients in the T12PR48 group who had had an extended rapid virologic response $(\mathrm{P}=0.22)$. $\mathrm{HCV}$ variants with decreased sensitivity to telaprevir were observed in the majority of patients in whom HCV RNA did not become undetectable, including the previously described variants with NS3/4A positions $36,54,155$, and $156 .{ }^{9}$ Follow-up of patients without a sustained virologic response showed that $55 \%$ of those with resistant variants at the time of treatment failure no longer had them (as detected by means of population sequencing) at their last visit (median follow-up, 43 weeks).

\section{SAFETY AND SIDE-EFFECT PROFILE}

In the overall treatment phase, $9 \%$ of patients had serious adverse events, anemia being the most common, reported in $2 \%$ of patients (Table 3 ). A total of $2 \%$ of patients in the T12PR24 group, as compared with 10\% of the patients in the T12PR48 group who had had 
an extended rapid virologic response, had serious adverse events $(P=0.005)$. Fatigue was the most common adverse event (68\%), followed by pruritus (51\%), nausea (47\%), anemia (39\%), headache (38\%), rash (37\%), insomnia (34\%), diarrhea (30\%), and influenza-like illness $(26 \%)$.

During the telaprevir treatment phase (i.e., the first 12 weeks), $7 \%$ of all patients discontinued all study drugs, with $1 \%$ attributable to rash events and $1 \%$ to anemia events. Twelve percent of patients discontinued telaprevir only, owing to adverse events, with $7 \%$ due to rash events and $2 \%$ to anemia events (Table 3).

Roughly two thirds of the observed rashes occurred in the first 8 weeks. Rashes were primarily eczematous in nature and were typically treated with topical medications (e.g., topical glucocorticoids or antihistamines), oral antihistamines, or both. Severe rash occurred in $5 \%$ of patients and was managed by means of discontinuation of telaprevir first, with continuation of peginterferon-ribavirin; if the rash did not improve within 7 days after telaprevir discontinuation, ribavirin (with or without peginterferon) was to be either interrupted or discontinued.

Severe anemia was reported in $6 \%$ of patients. During the treatment phase, 14 patients had low hemoglobin levels ( $<7 \mathrm{~g}$ per deciliter), classified as a grade 4 adverse event, with 11 of the 14 going on to have a sustained virologic response. A total of 32 patients received blood transfusions for treatment of anemia during the study; 7 patients (1\%) received an erythropoiesis-stimulating agent, and a sustained virologic response was achieved in 6 of these 7. The ribavirin dose was modified in $46 \%$ of patients because of decreased hemoglobin levels. A total of $73 \%$ of the patients whose ribavirin dose was not modified had a sustained virologic response, as did $68 \%$ and $75 \%$ of patients who had at least one dose reduction and at least one dose reduction and interruption, respectively. Among all study patients, $18 \%$ discontinued all study drugs because of adverse events, including $1 \%$ of the patients in the T12PR24 group and 12\% of the patients in the T12PR48 group who had had an extended rapid virologic response $(\mathrm{P}<0.001)$ (Table 3$)$.

\section{DISCUSSION}

We found that a 24-week treatment regimen of peginterferon-ribavirin, with telaprevir added for the first 12 weeks, was noninferior to a 48-week regimen of peginterferonribavirin, with telaprevir added for the first 12 weeks in patients with chronic infection with $\mathrm{HCV}$ genotype 1 who have not received treatment previously and who had an extended rapid virologic response. Nearly two thirds of the enrolled patients met this definition and were eligible for an abbreviated course of therapy. Thus, this study supports the concept of response-guided therapy. Relapse rates were low and were not significantly different between the 24-week group and the 48-week group. The sustained virologic response among all study patients was $72 \%$.

Telaprevir-based therapy guided by the presence or absence of an extended rapid virologic response selected for a population that demonstrated uniformly high rates of sustained virologic response regardless of race, ethnic group, or presence or absence of advanced fibrosis. There were high rates of response among blacks (60\%) and Hispanics or Latinos (67\%), in contrast with the historically poor responses in these subgroups reported in large published trials. The Viral Resistance to Antiviral Therapy of Chronic Hepatitis C study (VIRAHEP-C; ClinicalTrials.gov number, NCT00038974) reported a sustained virologic response of $28 \%$ among blacks receiving peginterferon-ribavirin. ${ }^{10}$ Response rates among patients with bridging fibrosis or cirrhosis were high; however, few patients had cirrhosis in our study, and further investigation is warranted. 
Virologic failure was frequently associated with the appearance of mutations related to resistance to telaprevir and other class-specific serine protease inhibitors. Follow-up of patients without a sustained virologic response in our study showed that $55 \%$ of patients who originally had resistant variants no longer had resistant variants at their last visit (median follow-up, 43 weeks). In addition, long-term follow-up of patients who had virologic failure in phase 2 telaprevir studies showed that in $89 \%$ of patients, telaprevirresistant variants were no longer detectable after a median follow-up time of 22 months, on the basis of population sequencing. ${ }^{11}$

Rates of drug discontinuation were similar to those in other clinical trials of telaprevir-based therapy $^{6-8}$ and were slightly higher than those observed in pivotal trials of peginterferonribavirin only. ${ }^{4,5}$ The overall incidences of rash (37\%) and severe rash (5\%) were similar to those in the 12-week telaprevir-based treatment groups in the ADVANCE (A New Direction in HCV Care: A Study of Treatment-Naive Hepatitis C Patients with Telaprevir) trial (NCT00627926) (37\% and 6\%, respectively). ${ }^{8}$ Although rash was common in our study, it was usually mild and was a relatively infrequent cause of discontinuation of all study medications. Our protocol differed from earlier phase 2 trials of telaprevir in that investigators were permitted to discontinue administration of telaprevir, in cases of severe or worsening rash, while maintaining the administration of peginterferon-ribavirin. Since most of these discontinuations occurred after $\mathrm{HCV}$ was undetectable (i.e., after 8 weeks of treatment), the discontinuation of telaprevir did not negatively affect the rate of sustained virologic response.

The incidence of anemia in our study (39\%) was similar to that in the 12-week telaprevirbased groups in ADVANCE (37\%). ${ }^{8}$ Rates of discontinuation of telaprevir and all study drugs owing to anemia-associated events were low. High rates of sustained virologic response were observed despite ribavirin-dose modifications, as well as in the small number of patients who received an erythropoiesis-stimulating agent.

In our phase 3 study, 24 weeks of peginterferon and ribavirin treatment was noninferior to 48 weeks of treatment for patients infected with HCV genotype 1 who had not previously received treatment and who had undetectable HCV RNA levels at weeks 4 and 12. This response-guided regimen resulted in a shorter treatment duration with high rates of sustained virologic response for approximately two thirds of treated patients. The treatment regimen was highly effective in patients with a historically poor treatment response, including blacks, patients with bridging fibrosis or cirrhosis, and patients with high HCV RNA levels.

Response-guided therapy also resulted in decreased rates of adverse events and treatment discontinuation among patients who received treatment over the shorter period.

\section{Supplementary Material}

Refer to Web version on PubMed Central for supplementary material.

\section{Acknowledgments}

Supported by Vertex Pharmaceuticals and Tibotec.

We thank the study coordinators, nurses, and patients involved in the study; the contract research organizations ICON and I3 for coordinating and implementing various aspects of the study; John Alam, M.D. (a former employee of Vertex Pharmaceuticals), for his contribution to the study design; and various Vertex Pharmaceuticals employees: Kristin Kleber for clinical project management; Tara L. Kieffer, Ph.D., and Doug J. Bartels, Ph.D., for virology analyses; Sandra Abensohn and Pamela Ryley for statistical programming; Concetta Marfella, Ph.D., for checking data accuracy and creating figures; and Mrudula Donepudi, Ph.D., for medical writing, editorial assistance, and coordination. 


\section{References}

1. Hepatitis C. Geneva: World Health Organization; 2011. http://www.who.int/vaccine_research/ diseases/hepatitis_c/en/

2. Bruno S, Stroffolini T, Colombo M, et al. Sustained virological response to interferon-alpha is associated with improved outcome in HCV-related cirrhosis: a retrospective study. Hepatology. 2007; 45:579-87. [PubMed: 17326216]

3. Morgan TR, Ghany MG, Kim HY, et al. Outcome of sustained virological responders with histologically advanced chronic hepatitis C. Hepatology. 2010; 52:833-44. [PubMed: 20564351]

4. Fried MW, Shiffman ML, Reddy KR, et al. Peginterferon alfa-2a plus ribavirin for chronic hepatitis C virus infection. N Engl J Med. 2002; 347:975-82. [PubMed: 12324553]

5. Manns MP, McHutchison JG, Gordon SC, et al. Peginterferon alfa-2b plus ribavirin compared with interferon alfa-2b plus ribavirin for initial treatment of chronic hepatitis $\mathrm{C}$ : a randomised trial. Lancet. 2001; 358:958-65. [PubMed: 11583749]

6. Hézode C, Forestier N, Dusheiko G, et al. Telaprevir and peginterferon with or without ribavirin for chronic HCV infection. N Engl J Med. 2009; 360:1839-50. [PubMed: 19403903]

7. McHutchison JG, Everson GT, Gordon SC, et al. Telaprevir with peginterferon and ribavirin for chronic HCV genotype 1 infection. N Engl J Med. 2009; 360:1827-38. Erratum, N Engl J Med 2009;361:1516. [PubMed: 19403902]

8. Jacobson IM, McHutchison JG, Dusheiko G, et al. Telaprevir for previously untreated chronic hepatitis C virus infection. N Engl J Med. 2011; 364:2405-6. [PubMed: 21696307]

9. Kieffer TL, Sarrazin C, Miller JS, et al. Telaprevir and pegylated interferon-alpha-2a inhibit wildtype and resistant genotype 1 hepatitis C virus replication in patients. Hepatology. 2007; 46:631-9. [PubMed: 17680654]

10. Conjeevaram HS, Fried MW, Jeffers LJ, et al. Peginterferon and ribavirin treatment in African American and Caucasian American patients with hepatitis C genotype 1. Gastroenterology. 2006; 131:470-7. [PubMed: 16890601]

11. Zeuzem S, Sulkowski MS, Zoulim F, et al. Long-term follow-up of patients with chronic hepatitis $\mathrm{C}$ treated with telaprevir in combination with peginterferon alfa- $2 \mathrm{a}$ and ribavirin: interim analysis of the EXTEND study. Hepatology. 2010; 52(Suppl 1):436A. abstract. [PubMed: 20683943] 


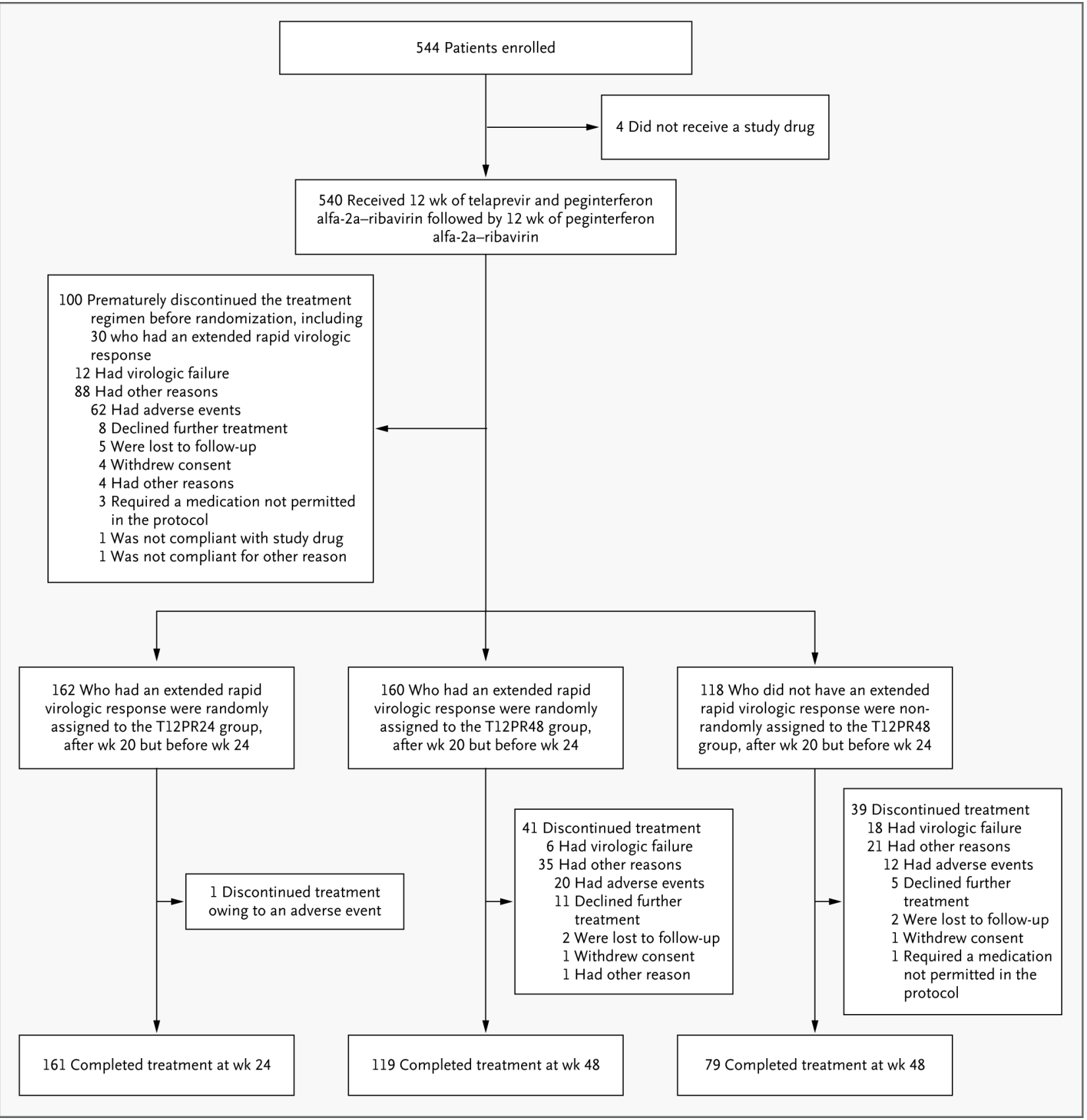

Figure 1. Enrollment, Randomization or Assignment, and Follow-up of the Study Patients Virologic failure was defined as an HCV RNA level greater than 1000 IU per milliliter at week 4 , a decline from baseline by less than $2 \log _{10}$ units in the level of detectable HCV RNA at week 12, or a detectable HCV RNA level at any time between weeks 24 and 36. 


\section{Table 1}

Baseline Characteristics of the Study Patients, According to Study Group.*

\begin{tabular}{|c|c|c|c|c|}
\hline Characteristic & $\begin{array}{c}\text { Randomly Assigned } \\
\text { to T12PR24 }(\mathrm{N}= \\
162)\end{array}$ & $\begin{array}{c}\text { Randomly Assigned } \\
\text { to T12PR48 }(\mathbf{N}= \\
160)\end{array}$ & $\begin{array}{l}\text { Nonrandomly Assigned } \\
\text { to } \mathrm{T12PR48}(\mathrm{N}=118)\end{array}$ & $\begin{array}{c}\text { Discontinued } \\
\text { Treatment before Wk } \\
20(\mathrm{~N}=100)\end{array}$ \\
\hline \multicolumn{5}{|l|}{ Age $-\mathrm{yr}$} \\
\hline Median & 51 & 50 & 51 & 52 \\
\hline Range & $22-70$ & $19-67$ & $20-63$ & $21-66$ \\
\hline \multicolumn{5}{|l|}{ Body-mass index ${ }^{\dagger}$} \\
\hline Median & 28 & 27 & 27 & 27 \\
\hline Range & $18-53$ & $19-49$ & $19-54$ & $19-44$ \\
\hline \multicolumn{5}{|l|}{ Distribution } \\
\hline$<25$ & $44(27)$ & $60(38)$ & $35(30)$ & $38(38)$ \\
\hline$\geq 25$ to $<30$ & $56(35)$ & $51(32)$ & $49(42)$ & $32(32)$ \\
\hline 230 & $61(38)$ & $49(31)$ & $34(29)$ & $30(30)$ \\
\hline Missing data & $1(1)$ & 0 & 0 & 0 \\
\hline Male sex — no. (\%) & $104(64)$ & $97(61)$ & $70(59)$ & $54(54)$ \\
\hline \multicolumn{5}{|l|}{ Race - no. $(\%)^{\psi^{*}}$} \\
\hline White & $135(83)$ & $131(82)$ & $86(73)$ & $75(75)$ \\
\hline Black & $17(10)$ & $17(11)$ & $20(17)$ & $19(19)$ \\
\hline Other & $10(6)$ & $12(8)$ & $12(10)$ & $6(6)$ \\
\hline \multicolumn{5}{|c|}{ Hispanic or Latino ethnic group — no. $(\%)$} \\
\hline Yes & $18(11)$ & $11(7)$ & $8(7)$ & $17(17)$ \\
\hline No & $140(86)$ & $146(91)$ & $105(89)$ & $82(82)$ \\
\hline Missing data & $4(2)$ & $3(2)$ & $5(4)$ & $1(1)$ \\
\hline \multicolumn{5}{|l|}{ HCV genotype 1 subtype - no. $(\%)^{\xi}$} \\
\hline 1a & $115(71)$ & $117(73)$ & $84(71)$ & $72(72)$ \\
\hline $1 b$ & $46(28)$ & $43(27)$ & $33(28)$ & $27(27)$ \\
\hline Unknown & $1(1)$ & 0 & $1(1)$ & $1(1)$ \\
\hline HCV RNA $\log _{10}-\mathrm{IU} / \mathrm{ml} I /$ & $6.3 \pm 0.9$ & $6.4 \pm 0.7$ & $6.7 \pm 0.6$ & $6.4 \pm 0.7$ \\
\hline $\begin{array}{l}\text { HCV RNA level } \geq 800,000 \mathrm{IU} / \mathrm{ml} \\
- \text { no. }(\%)^{q /}\end{array}$ & $124(77)$ & $126(79)$ & $108(92)$ & $87(87)$ \\
\hline \multicolumn{5}{|c|}{ Stage of fibrosis and cirrhosis - no. (\%) } \\
\hline None or minimal fibrosis & $46(28)$ & $48(30)$ & $27(23)$ & $26(26)$ \\
\hline Portal fibrosis & $78(48)$ & $79(49)$ & $49(42)$ & $38(38)$ \\
\hline Bridging fibrosis & $20(12)$ & $21(13)$ & $30(25)$ & $17(17)$ \\
\hline Cirrhosis & $18(11)$ & $12(8)$ & $12(10)$ & $19(19)$ \\
\hline
\end{tabular}

* Plus-minus values are means \pm SD. None of the characteristics differed significantly between the randomized groups $(\mathrm{P}>0.05$ for all comparisons). Patients received telaprevir ( $750 \mathrm{mg}$ every 8 hours) for 12 weeks, as well as peginterferon alfa-2a (180 $\mu \mathrm{g}$ per week) and ribavirin (1000 or $1200 \mathrm{mg}$ per day, according to body weight) for a total of either 24 weeks (T12PR24) or 48 weeks (T12PR48). Patients who had an extended rapid virologic response were randomly assigned to either the T12PR24 group or the T12PR48 group. Patients who did not have an 
extended rapid virologic response were nonrandomly assigned to the T12PR48 group. The remaining patients discontinued the treatment regimen before week 20 or nonrandom assignment. HCV denotes hepatitis $\mathrm{C}$ virus.

The body-mass index is the weight in kilograms divided by the square of the height in meters.

"Race and ethnic group were self-reported and were not mutually exclusive. The "other" race category included patients self-identifying as Asian, American Indian or Alaska Native, Native Hawaiian or other Pacific Islander, or other self-reported races, as well as 13 patients for whom local regulations did not permit asking about race or ethnic group.

$\xi_{\mathrm{HCV}}$ genotype and subtype were ascertained by means of line-probe assay (Inno-LiPA, Innogenetics).

I/ HCV RNA levels were measured with the use of the COBAS TaqMan HCV assay (Roche Molecular Systems), which has a lower limit of quantification of $25 \mathrm{IU}$ per milliliter and a lower limit of detection of approximately 10 to $15 \mathrm{IU}$ per milliliter. 


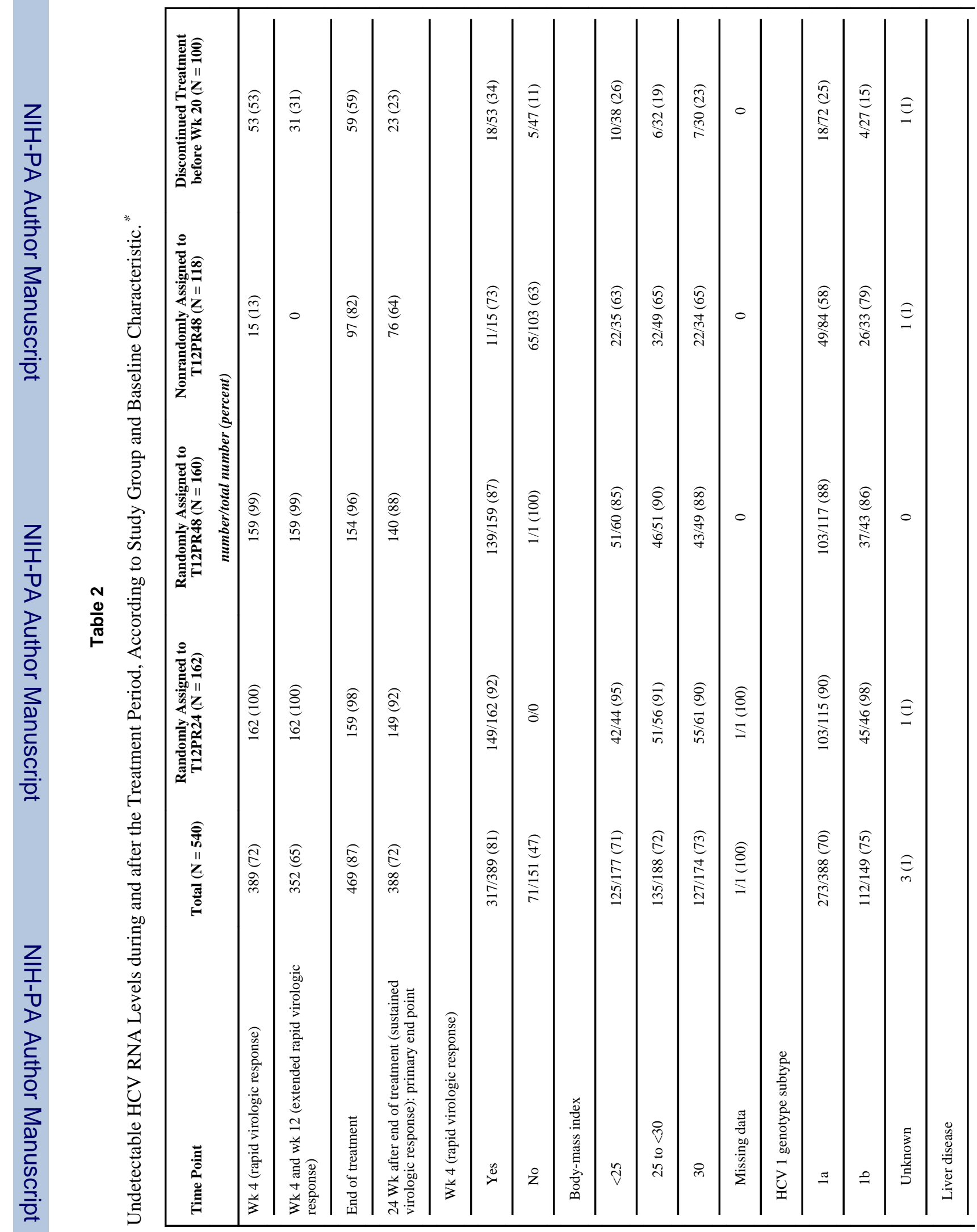




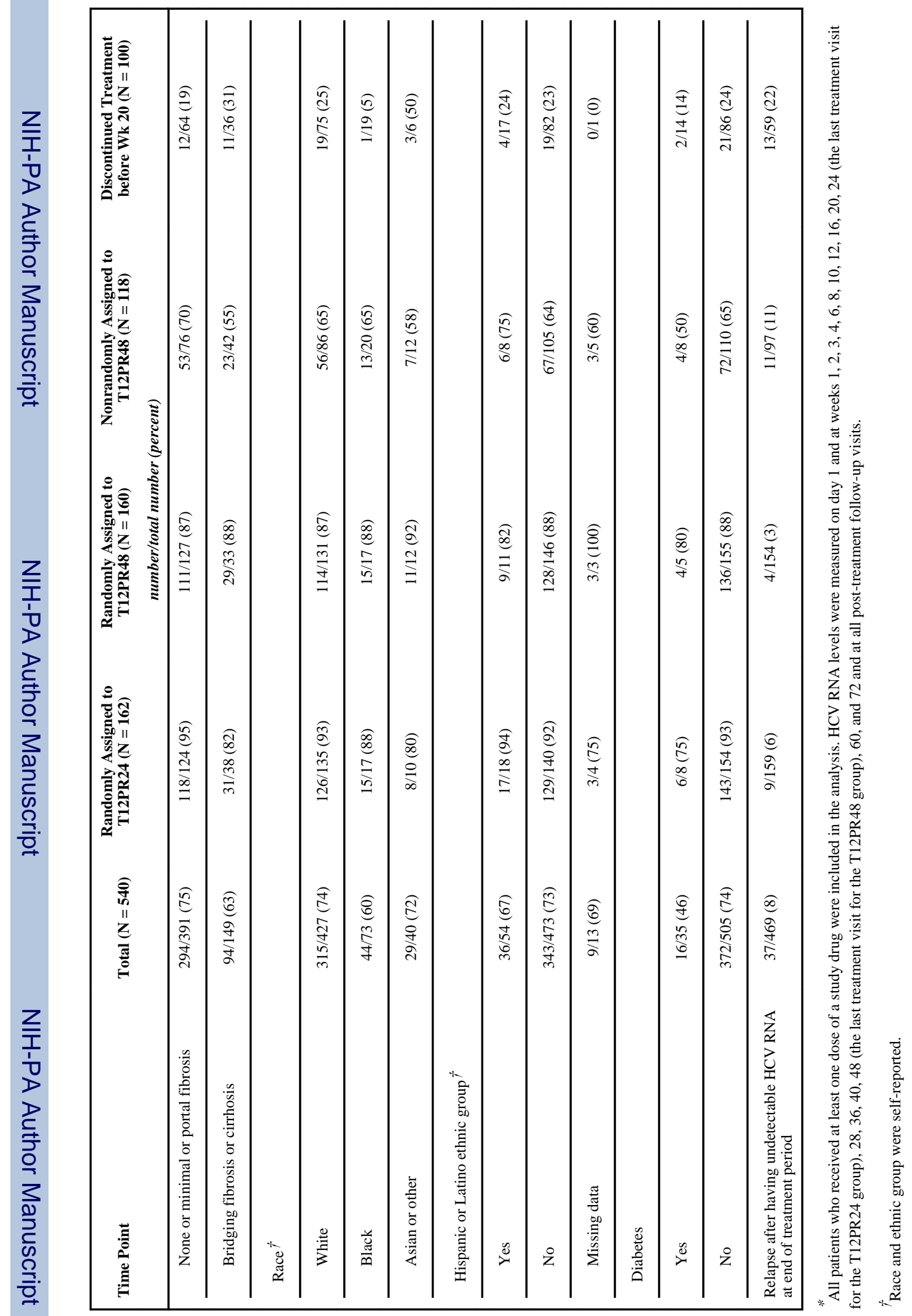

N Engl J Med. Author manuscript; available in PMC 2013 October 28. 


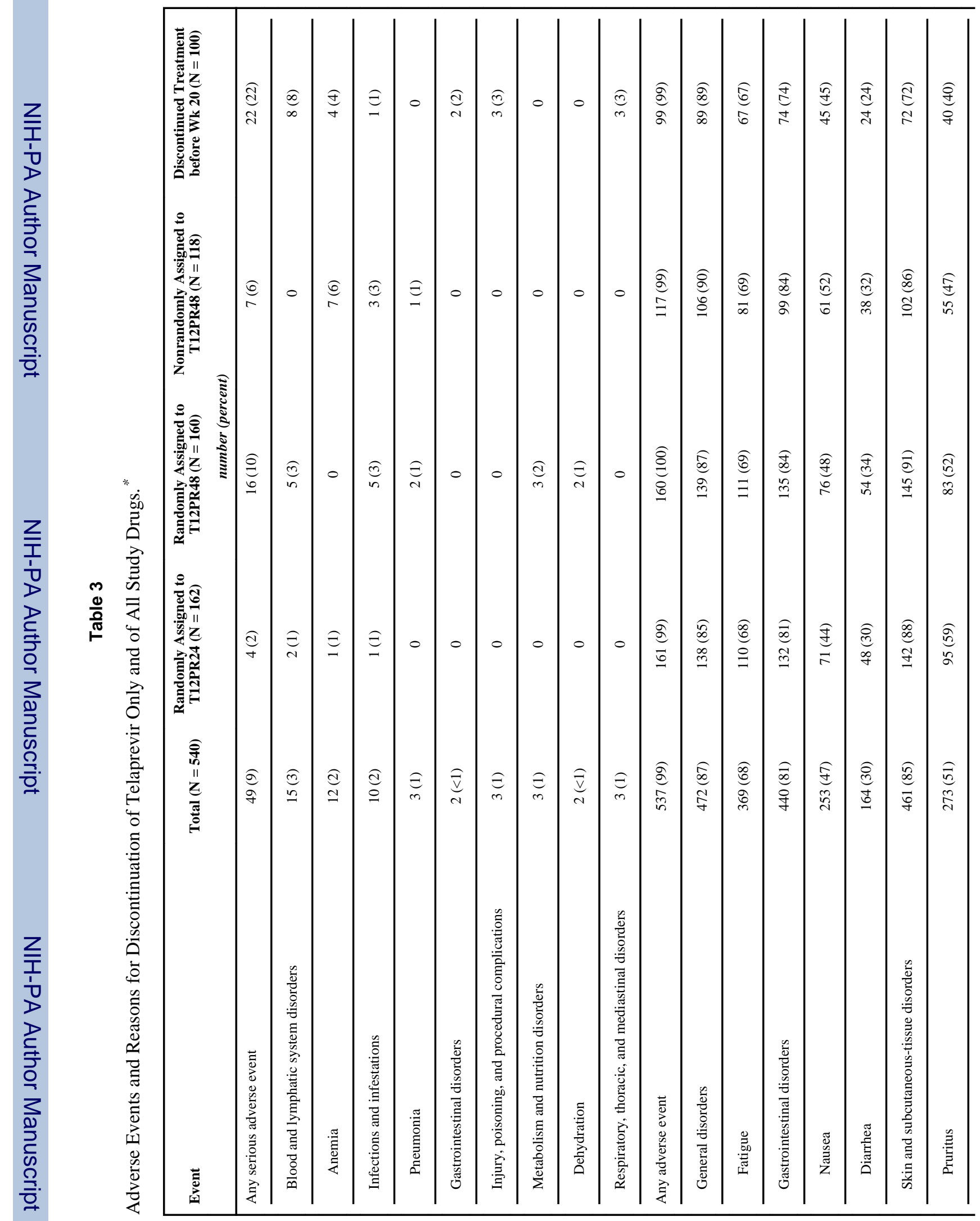

NEngl J Med. Author manuscript; available in PMC 2013 October 28. 


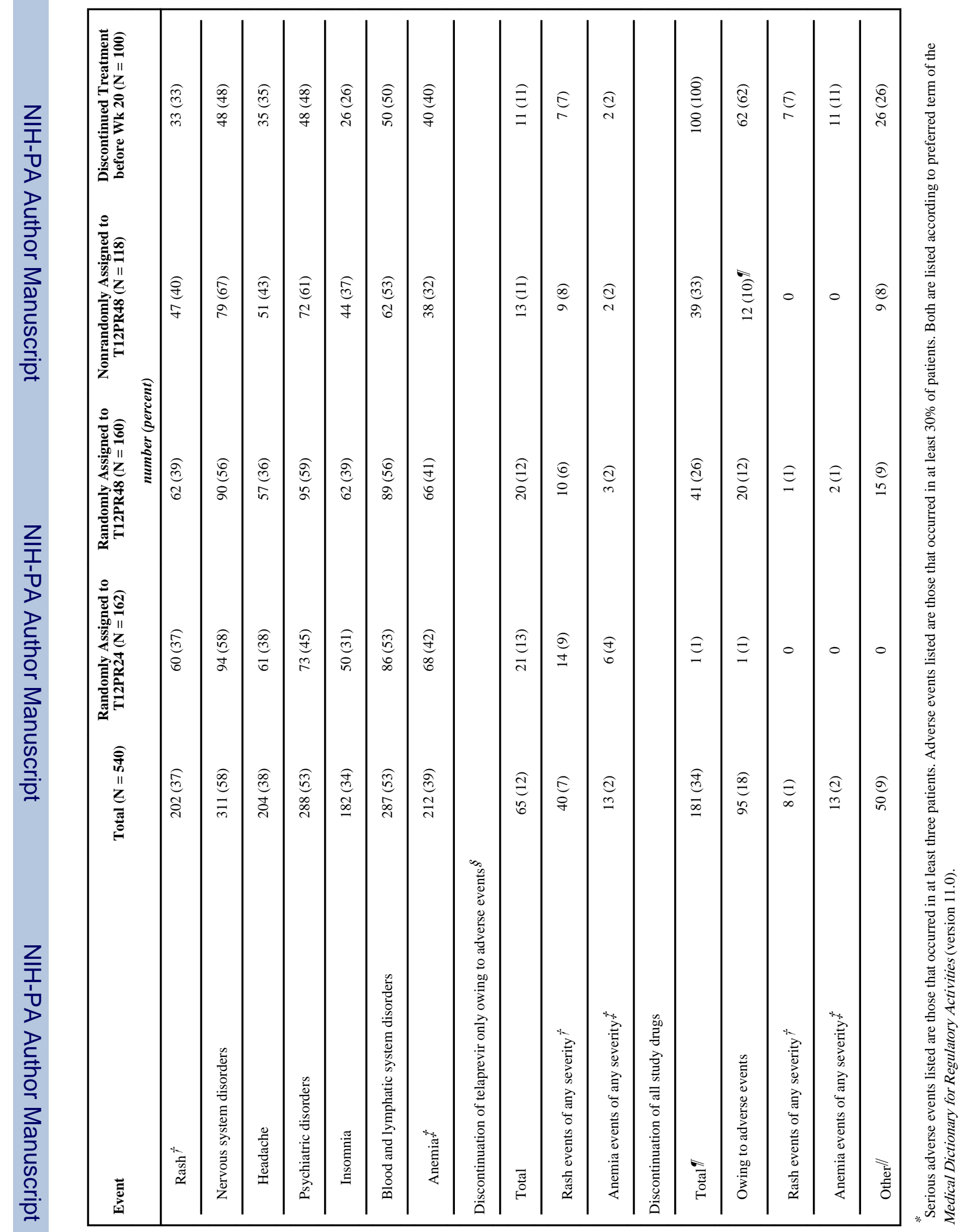




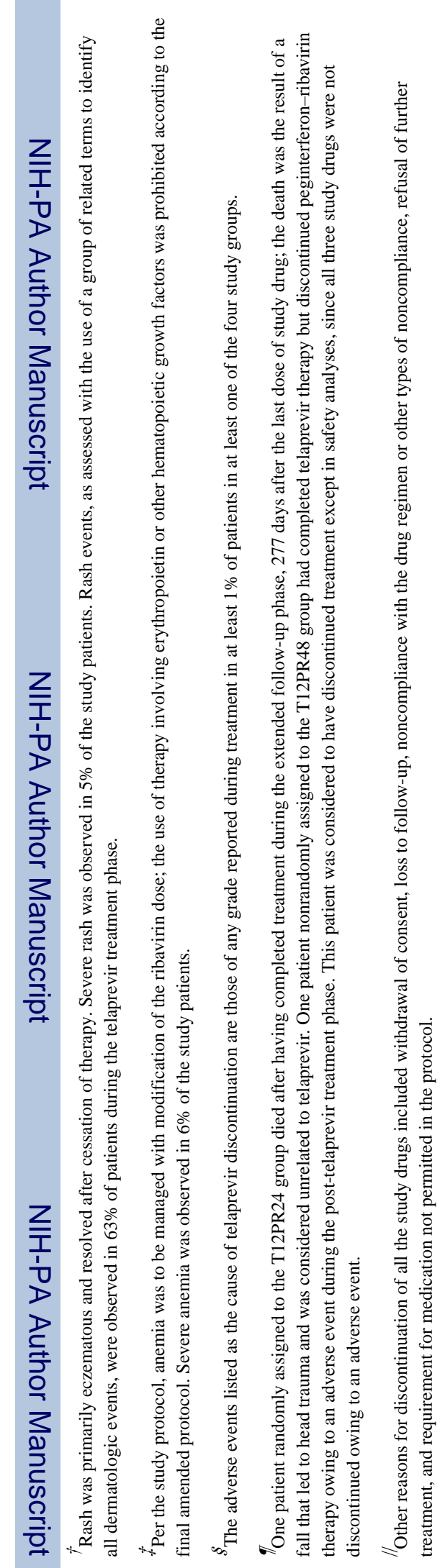

N Engl J Med. Author manuscript; available in PMC 2013 October 28. 